\title{
Computing the Narumi-Katayama indices and its modified version of some nanostar dendrimers
}

\author{
Islam Goli Farkousha ${ }^{-}$Mehdi Alaeiyan ${ }^{b, *}$ | Mohammad Maghasedia
}

aDepartment of Mathematics, Karaj Branch, Islamic Azad University, Karaj, Iran

${ }^{b}$ Department of Mathematics, Iran University of Science and Technology, Narmak, Tehran 16844, Iran
Dendrimers are the highly branched organic macromolecules with successive layers or generations of branch units surrounding a central core. In mathematical chemistry, a particular attention has been given to degree-based graph invariant. The Narumi-Katayama index and its modified version of a graph $\mathrm{G}$, denoted by $\mathrm{NK}(\mathrm{G})$ and $\mathrm{NK}^{*}(\mathrm{G})$ are equal to the product of the degrees of the vertices of $G$. In this work we calculated the Narumi-Katayama Indices and its Modified version for some families of dendrimers such as $N S_{1}[n], N S_{2}$ [n] and $N S_{3}[n]$. The exact formulas of the Narumi-Katayama and Modified Narumi-Katayama indices of these dendrimers nano structures are presented in this paper.

*Corresponding Author:

Mehdi Alaeiyan

Email: alaeiyan@iust.ac.ir

Tel.: +989123907865

\section{Introduction}

Chemists are equipped with a number of useful tools known as merging branch of graph theory (chemical graph theory), molecular topological descriptors, indices and molecular topological polynomials. Many chemical structures, and chemical compounds are usually modeled by a molecular graph to analyze the underlying theoretical properties. Several articles contributed to determine the topological indices of special molecular graphs [13-15].

Dendrimers are a new class of polymeric materials. They are highly branched, monodisperse macromolecules. The structure of these materials has a great impact on their physical and chemical properties. As a result of their unique behavior dendrimers are suitable for awide range of biomedical and industrial applications [2].

The nanostar dendrimers is a part of a new group of macromolecules that seem photon funnels just like artificial antennas and also is a great resistant of photo bleaching [2]. Recently some researchers investigated the mathematical properties of this nano structures [1-12].

A molecular graph is a pictorial diagram of the structural formula of a chemical structure in terms of graph theory, where the vertices represent the atoms of the given chemical compound and the edges represent the chemical bonds between the atoms. We consider the molecular graph $\mathrm{G}=(\mathrm{V}, \mathrm{E})$ with the vertex set $V(G)$ and the edge set $E(G)$ is a graph whose vertices denote atoms and edges denote bonds between the atoms of any underlying chemical structure [3,16-26]. The degree of a vertex $v$, denoted by $d_{G}(v)$, is the simplicity, let $d_{G}(v)=d_{v}$.

A topological index is a single number, derived following a certain rule, which can be used to characterize the molecule [5]. Usage of topological indices in biology and chemistry began in 1947 when chemist Harold Wiener [4] introduced Wiener index to demonstrate correlations between chemical properties of organic compounds and the index of their molecular graphs. Wiener originally defined his index (W) on number of edges that are incident to it. For 
trees and studied its use for correlation of chemical properties of alcohols, amines and their analogous compounds [4].

A topological list $\operatorname{Top}(\mathrm{G})$ of graph $\mathrm{G}$ is a number with the property that, for each diagram $\mathrm{H}$ isomorphic to chart $\mathrm{G}$. $\operatorname{Top}(H)=\operatorname{Top}(G)$. In $[3,6]$ authors studied some properties of Narumi-Katayama indices and its modified defined as follows:

$$
\begin{aligned}
& N K(G)=\prod_{u \in V(G)} d_{u} \\
& N K^{*}(G)=\prod_{u \in V(G)} d_{u}{ }^{d_{u}}
\end{aligned}
$$

Several studies contributed to determine the topological indices of some families of dendrimers [1, 7-9]. In this work, we compute the Narumi-Katayama index and its modified for some families of dendrimers.

\section{Main Results}

In this section, we compute the NarumiKatayama indices and its modified of some nanostar dendrimers. Suppose that the first kind of nanostar $N S_{1}[n]$ with n growth stages. The molecular graph of $N S_{1}[1]$ and $N S_{1}[3]$ are shown in Figures 1 and 2 and $N S_{2}[3]$ and $\mathrm{NS}_{3}$ [2] are shown in Figures 3 and 4. Note that the number $\mathrm{C}_{6}$ (hexagonal) in the graph $N S_{1}[n]$ is $3 \times 2^{n-2}-5$ for each step $n \in \mathrm{N} \bigcup\{0\}$.

Theorem 2.1. Consider $N S_{1}[n]$ be the Nanostar with $\mathrm{n}$ growth of stages and $n \in \mathrm{N} \bigcup\{0\}$. Then the Narumi-Katayama indices and its modified of $N S_{1}[n]$ are given by

$$
\begin{aligned}
& \text { i) } N K\left(N S_{1}[n]\right)=2^{18 \times 2^{n}-1} \times 3^{6 \times 2^{n}-3}, \\
& \text { ii) } N K^{*}\left(N S_{1}[n]\right)=2^{36 \times 2^{n}+2} \times 3^{18 \times 2^{n}-9} .
\end{aligned}
$$

Proof. Let $N S_{1}[n]$ be the Nanostar with $\mathrm{n}$ growth of stages and $n \in \mathrm{N} \bigcup\{0\}$. The number of vertices and edges in $N S_{1}[n]$ are $24 \times 2^{n-4}$ and $27 \times 2^{n-5}$. The vertex set $V\left(N S_{1}[n]\right)$ and the edge set $E\left(N S_{1}[n]\right)$ are equal to $24 \times 2^{n-4}$ and $27 \times 2^{n-5}$. The vertex set $V\left(N S_{1}[n]\right)$ can be divided into four vertex partitions based on degrees of vertices $V_{1}, V_{2}, V_{3}, V_{4}$ where $V_{i}=\left\{u \mid u \in V\left(N S_{1}[n]\right), d_{u}=\mathrm{i}\right\} ; 1 \leq \mathrm{i} \leq 4$. The number of vertices $V_{1}\left(N S_{1}[n]\right), V_{4}\left(N S_{1}[n]\right)$ are 1 , moreover we have:

$\left\{\begin{array}{c}V_{1}+2 V_{2}+3 V_{3}+4 V_{4}=2 E \\ V_{1}+V_{2}+V_{3}+V_{4}=V\end{array}\right.$

Therefore, by solving the above equation, the number of vertices in $V_{2}\left(N S_{1}[n]\right)$, and $V_{3}\left(N S_{1}[n]\right)$, are $18 \times 2^{n-3}$ and $6 \times 2^{n-3}$. Now by using (1.1) and (1.2), we have:

$$
\begin{aligned}
& N K\left(N S_{1}[n]\right)=\prod_{u \in V\left(N S_{1}[n]\right)} d_{u}=\prod_{\substack{\left.u_{i} \in V_{i}\left(N S_{[}[n]\right) \\
1 \leq i \leq \leq 4\right)}} d_{u_{i}} \\
& =1^{\left|V_{1}\left(N S_{1}[n]\right)\right|} \times 2^{\left|V_{2}\left(N S_{1}[n]\right)\right|} \times 3^{\left|V_{3}\left(N S_{1}[n]\right)\right|} \times 4^{\left|V_{4}\left(N S_{1}[n]\right)\right|} \\
& =1^{1} \times 2^{18 \times n^{n}-3} \times 3^{6 \times 2^{n}-3} \times 4^{1}=2^{18 \times 2^{n}-1} \times 3^{6 \times 2^{n}-3} .
\end{aligned}
$$

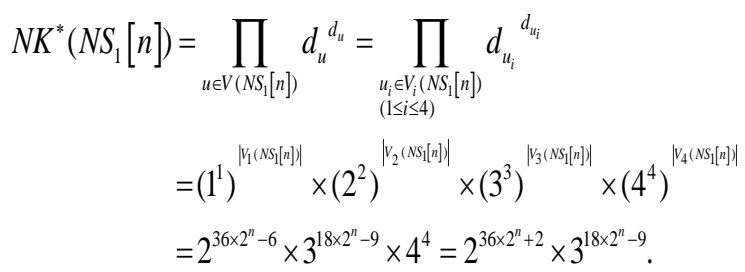

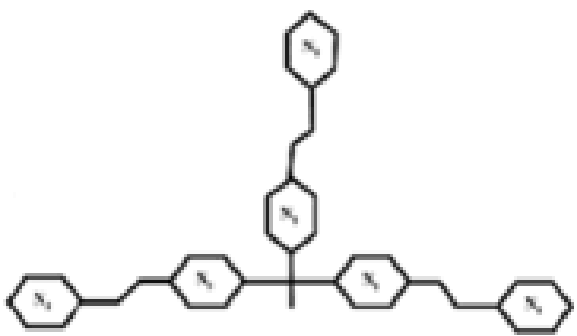

FIGURE 1 The core of $N S_{1}[1]$

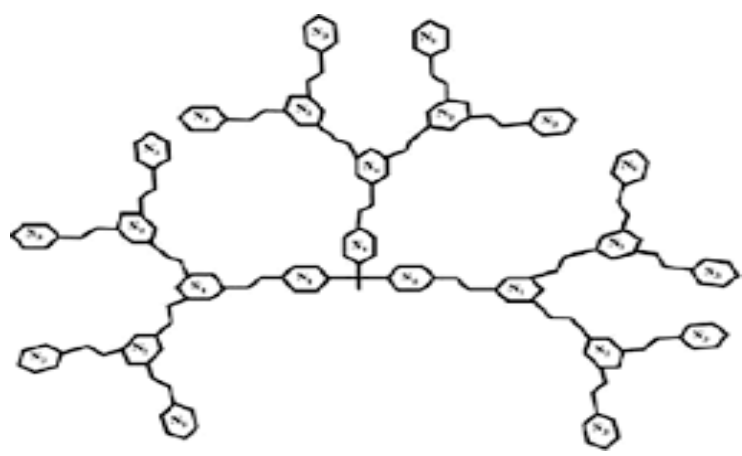

FIGURE 2 The core of $N S_{1}[3]$

Example 2.1. Consider nanostare dendrimer $N S_{1}[1]$ with $\mathrm{n}$ growth of stages and $n \in \mathrm{N} \bigcup\{0\}$ is shown in Figure 1. By theorem 2.1 , the number of vertices in $N S_{1}[1]$ is 40 and its edges is 49 . The vertex partitions $V_{1}\left(N S_{1}[1]\right), \quad V_{2}\left(N S_{1}[1]\right), \quad V_{3}\left(N S_{1}[1]\right), \quad$ and 
$V_{4}\left(N S_{1}[1]\right)$ contains respectively $1,33,9$ and 1 vertices. Then

$$
\begin{aligned}
& N K\left(N S_{1}[1]\right)=\prod_{u \in V\left(N S_{1}[1]\right)} d_{u} \\
& =\prod_{u_{i} \in V_{i}\left(N S_{1}[1]\right)} d_{u_{i}},(1 \leq i \leq 4) \\
& =1^{\mid V_{1}\left(N S_{1}[1]\right\rangle} \times 2^{\left|V_{2}\left(N S_{1}[1]\right)\right|} \times 3^{\left|V_{3}\left(N S_{1}[1]\right)\right|} \times 4^{\mid V_{4}\left(N S_{1}[1] \mid\right.} \\
& =1^{1} \times 2^{33} \times 3^{9} \times 4^{1}=2^{35} \times 3^{9} \text {. } \\
& N K^{*}\left(\mathrm{NS}_{1}[1]\right)=\prod_{u \in V\left(\mathrm{NS}_{1}[1]\right)} d_{u}^{d_{u}} \\
& =\prod_{u_{i} \in V_{i}\left(N S_{1}[1]\right)} d_{u_{i}}^{d_{u_{i}}},(i=1,2,3,4) \\
& =\left(1^{1}\right)^{\mid V_{1}\left(N S_{S}[1]\right)} \times\left(2^{2}\right)^{\mid V_{2}\left(N S_{S}[1]\right)} \times\left(3^{3}\right)^{\left|V_{3}\left(N S_{[}[]\right]\right|} \times\left(4^{4}\right)^{\mid V_{4}\left(N N_{S}[1] \mid\right.} \\
& =2^{64} \times 3^{27} \times 4^{4}=2^{74} \times 3^{27} \text {. }
\end{aligned}
$$

Theorem 2.2. Consider $\mathrm{NS}_{2}[n]$ be the nanostare with $\mathrm{n}$ growth of stages and $n \in \mathrm{N} \cup\{0\}$. Then the Narumi-Katayama Indices and its Modified of $N S_{2}[n]$ are given by i) $N K\left(N S_{2}[n]\right)=2^{12 \times 2^{n}-2} \times 3^{4 \times 2^{n}-2}$, ii) $N K^{*}\left(N S_{2}[n]\right)=2^{24 \times 2^{n}-4} \times 3^{12 \times 2^{n}-6}$.

Proof. Let $N S_{2}[n]$ be the Nanostar. The number of vertices and edges in $N S_{2}[n]$ are $16 \times 2^{n-4}$ and $18 \times 2^{n}-5$ respectively, The vertex set $\left.V\left(N S_{2}[n]\right)\right)$ is divided into two vertex partitions based on degrees of vertices. The first vertex Partition $V_{1}\left(N S_{2}[n]\right)$ where $d_{u_{1}}=2, u_{1} \in V_{1}\left(N S_{2}[n]\right)$, the second vertex partition $\quad V_{2}\left(N S_{2}[n]\right), \quad$ where $d_{u_{2}}=3, u_{2} \in V_{2}\left(N S_{2}[n]\right)$. Moreover we have:

$$
\left\{\begin{array}{c}
2 V_{1}+3 V_{2}=2 E \\
V_{1}+V_{2}=V
\end{array}\right.
$$

Therefore, by solving the above equation the number of vertices in $V_{1}\left(N S_{2}[n]\right)$ and $V_{2}\left(N S_{2}[n]\right)$ are $12 \times 2^{n-2}$ and $4 \times 2^{n-2}$ respectively. Now by using (1.1) and (1.2), we have:

$$
\begin{aligned}
N K\left(N S_{2}[n]\right)= & \prod_{u \in V\left(N S_{2}[n]\right)} d_{u} \\
= & \prod_{u_{1} \in V_{1}\left(N S_{2}[n]\right)} d_{u_{1}} \times \prod_{u_{2} \in V_{2}\left(N S_{2}[n]\right)} d_{u_{2}} \\
= & 2^{\left|V_{1}\left(N S_{2}[n]\right)\right|} \times 3^{\left|V_{2}\left(N S_{2}[n]\right)\right|}=2^{12 \times 2^{n}-2} \times 3^{4 \times 2^{n}-2} .
\end{aligned}
$$

$$
\begin{aligned}
N K^{*}\left(N S_{2}[n]\right) & =\prod_{u \in V\left(N S_{2}[n]\right)} d_{u}^{d_{u}} \\
& =\prod_{u_{1} \in V_{1}\left(N S_{2}[n]\right)} d_{u_{1}}^{d_{u_{1}}} \times \prod_{u_{2} \in V_{2}\left(N S_{2}[n]\right)} d_{u_{2}}^{d_{u_{2}}} \\
& =\left(2^{2}\right)^{\mid V_{1}\left(N S_{2}[n] \mid\right)} \times\left(3^{3}\right)^{\mid V_{2}\left(N S_{2}[n] \mid\right.} \\
& =\left(2^{2}\right)^{12 \times 2^{n}-2} \times\left(3^{3}\right)^{4 \times 2^{n}-2}=2^{24 \times 2^{n}-4} \times 3^{12 \times 2^{n}-6} .
\end{aligned}
$$

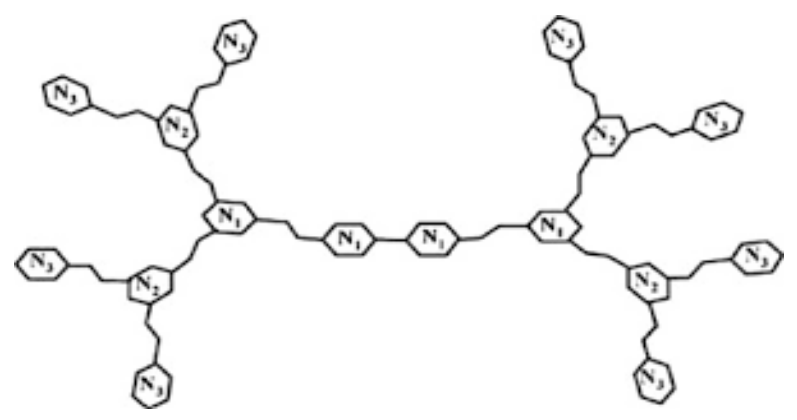

FIGURE 3 Nanostar dendrimer with 3-growth stages $N S_{2}[3]$

Example 2.2. Consider nanostare dendrimer $N S_{2}$ [3] with $\mathrm{n}$ growth of stages and $n \in \mathrm{N} \bigcup\{0\}$ is shown in Figure 4. Then, the number of vertices in $\mathrm{NS}_{2}[3]$ is 124 and its edges is 139 . The vertex partition in $V_{1}\left(N S_{2}[3]\right)$ contains 94 vertices $u_{1}$ whit degree 2 and the vertex partition in $V_{2}\left(N S_{2}[n]\right)$ contains 30 vertices $u_{2}$ whit degree 3 . Then

$$
\begin{aligned}
N K\left(N S_{2}[3]\right) & =\prod_{u \in V\left(N S_{2}[3]\right)} d_{u} \\
& =\prod_{u_{1} \in V_{1}\left(N S_{2}[3]\right)} d_{u_{1}} \times \prod_{u_{2} \in V_{2}\left(N S_{2}[3]\right)} d_{u_{2}} \\
& =2^{\mid V_{1}\left(N S_{2}[3]||\right.} \times 3^{\mid V_{2}\left(N S_{2}[3] \mid\right.}=2^{94} \times 3^{30} . \\
N K^{*}\left(N S_{2}[3]\right) & =\prod_{u \in V\left(N S_{2}[3]\right)} d_{u}^{d_{u}} d_{u_{1} \in V_{1}\left(N S_{2}[3]\right)} d_{u_{1}} \times \prod_{u_{2} \in V_{2}\left(N S_{2}[3]\right)} d_{u_{2}} d_{u_{2}} \\
& =\left(2^{2}\right)^{\mid V_{1}\left(N S_{2}[3] \mid\right.} \times\left(3^{3}\right)^{\mid V_{2}\left(N S_{2}[3] \mid\right.}=2^{188} \times 3^{60} .
\end{aligned}
$$

Theorem 2.3. Consider $N S_{3}[n]$ be the Nanostar with $\mathrm{n}$ growth of stages and $n \in \mathrm{N} \bigcup\{0\}$. Then the Narumi-Katayama Indices and its Modified of $N S_{3}[n]$ are given by $N K\left(N S_{3}[n]\right)=2^{12 \times 2^{n}-6} \times 3^{6 \times 2^{n}-6}$,
$N K^{*}\left(N S_{3}[n]\right)=2^{24 \times 2^{n}-12} \times 3^{18 \times 2^{n}-18}$. 
Proof. Similar to the proof of Theorem 2.2.

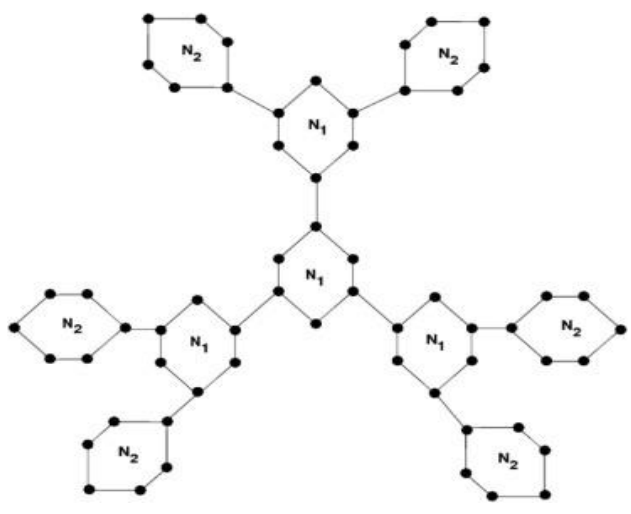

FIGURE 4 Nanostar dendrimer with 2-growth stages $N S_{3}[2]$

Example 2.3. Consider Nanostar dendrimer $N S_{3}$ [2] with $n$ growth of stages and $n \in \mathrm{N} \bigcup\{0\}$ is shown in Figure 2. Then, the number of vertices in $N S_{3}[n]$ is 60 and its edges is 69 . The vertex partition $V_{1}\left(N S_{3}[2]\right)$ contains 42 vertices $u_{1}$ whit degree 2 and the vertex partition $V_{2}\left(\mathrm{NS}_{3}[2]\right)$ contains 18 vertices $u_{2}$ whit degree 3 . Then

$$
\begin{aligned}
N K\left(N S_{3}[2]\right) & =\prod_{u \in V\left(N S_{3}[2]\right)} d_{u} \\
& =\prod_{u_{1} \in V_{1}\left(N S_{3}[2]\right)} d_{u_{1}} \times \prod_{u_{2} \in V_{2}\left(N S_{3}[2]\right)} d_{u_{2}} \\
& =2^{\mid V_{1}\left(N S_{3}[2] \mid\right.} \times 3^{\left|V_{2}\left(N S_{3}[2]\right)\right|}=2^{42} \times 3^{18} . \\
N K^{*}\left(N S_{3}[2]\right) & =\prod_{u \in V\left(N S_{3}[2]\right)} d_{u}^{d_{u}} \\
& =\prod_{u_{1} \in V_{1}\left(N S_{3}[2]\right)} d_{u_{1}}^{d_{u_{1}}} \times \prod_{u_{2} \in V_{2}\left(N S_{3}[2]\right)} d_{u_{2}} d_{u_{2}} \\
& =\left(2^{2}\right)^{\left|V_{1}\left(N S_{3}[2]\right)\right|} \times\left(3^{3}\right)^{\mid V_{2}\left(N S_{3}[2] \mid\right.}=2^{84} \times 3^{54} .
\end{aligned}
$$

\section{Conclusion}

Molecular topology (or topological indices) has widely demonstrated its high performance in the discovery and design of new drugs. In this research study, we contributed to a better knowledge of molecular topology among mathematicians. Moreover: molecular topology can be employed to look for drugs that heal, in principle, any disease, based on the structural information provided by known active compounds. In this research study, we determined the Narumi-Katayama indices and modified for some families of nanostar dendrimers such as $N S_{1}[n], N S_{2}[n]$ and $N S_{3}[n]$. In the future, we are interested to study and compute the topological indices of various families of dendrimers which will be quite useful in understanding their underlying topologies.

\section{Acknowledgements}

The authors are thankful to the reviewers for their valuable comments and suggestions which improved the presentation of the paper.

\section{Orcid:}

Mehdi Alaeiyan: https://orcid.org/0000-00032185-5967

Islam Goli Farkoush: https://orcid.org/00000002-1110-8629

\section{References}

[1] A.R. Ashrafi, M. Mirzargar, Indian J. Chem., 2008, 47A, 538-541.

[2] B. Klajnert, M. Bryszewska, Acta Biochim. Polonica., 2001, 48, 199-208.

[3] H. Narumi, M. Katayama. Mem. Fac. Engin. Hokkaido Univ., 1984, 16, 209-214.

[4] H. Wiener, J Amer. Chem. Soc., 1947, 69, 1, 17-20.

[5] I. Gutman, M. Ghorbani. Appl. Math. Lett., 2012, 25, 1435-1438.

[6] I. Goli Farkoush, M. Alaeiyan, M. Maghasedi. J Dis. Math. Sci. Crypt., 2019, 22, 1189-1197.

[7] N. Dorostic, A. Iranmanesh, M.V. Diudea, Math Commun. Match. Comput. Chem., 2009, 062, 389-395.

[8] N.E. Arif, R. Hasni, S. Alikhani, Dig. J. Nano. Biostr., 2011, 6, 1551-1556.

[9] S. Alikhani, M.A. Iranmanesh, J. Comput. Theor. Nanosc., 2010, 7, 2314-2316. 
[10] A. Iranmanesh, N.A. Gholami, MATCH Commun. Math. Comput. Chem., 2009, 62, 371-379.

[11] M. Mirzagar, MATCH Commun. Math. Comput. Chem., 2009, 62, 363-370.

[12] H. Yousefi-Azari, A.R. Ashrafi, A. Baharin, Y. Yazdani, Asian J. Chem., 2008, 20, 15-20.

[13] W. Gao, L. Liang, Y. Gao. Energy Edu. Sci Tec: Part A., 2014, 32, 8961-8970.

[14] W. Gao, L. Liang, Y. Gao. Bio Technology: An Indian J., 2014, 10, 3837-3845.

[15] W. Gao, M.R. Farahani. J Adv Chem., 2015, 12, 3934-3939.

[16] E.G.A. Gomaa, M.A. Berghout, M.R. Moustafa, F.M. El Taweel, H.M. Farid, Prog. Chem. Biochem. Res., 2018, 1, 19-28.

[17] M. Nabati, V. Bodaghi-Namileh, S. Sarshar, Prog. Chem. Biochem. Res., 2019, 2, 108-119.

[18] M.E. Khan, A.S. Adeiza, T.A. Tor-Anyiin, A. Alexander, Prog. Chem. Biochem. Res., 2019, 2, 68-73.

[19] J.-B. Liu, A.Q. Baig, M. Imran, W. Khalid, M. Saeed, M.R. Farahani, Eurasian Chem. Commun., 2020, 2, 672-679.

[20] M. Imran, S.A. Ul Haq Bokhary, S. Manzoor, M.K. Siddiqui, Eurasian Chem. Commun., 2020, 2, 680-687.
[21] Y. Gao, M.R. Farahani, W. Nazeer, Chem. Methodol., 2018, 3, 39-45.

[22] D.X. Li, J.-B. Liu, M. Rezaei, M.R. Farahani, J. Comput. Theor. Nanosci., 2016, 13, 91369139.

[23] W. Gao, M.R. Farahani, J. Dis. Math. Sci. Crypt., 2017, 20, 515-523.

[24] W. Gao, L. Shi, M.R. Farahani, IAENG Int. J. App. Math., 2016, 46, 168-186.

[25] Z. Foruzanfar, M.K. Jamil, M.R. Farahani, M. Imran, X. Zhang, Int. J. Pur. App. Math., 2017, 117, 173-183.

[26] M.R. Farahani, A.Q. Baig, W. Sajjad, J. Math. Nanosci., 2017, 7, 23-28.

How to cite this article: Islam Goli Farkoush, Mehdi Alaeiyan*, Mohammad Maghasedi. Computing the NarumiKatayama indices and its modified version of some nanostar dendrimers. Eurasian Chemical Communications, 2020, 2(7), 771775.

Link:

http://www.echemcom.com/article_10594 2.html

Copyright (C) 2020 by SPC (Sami Publishing Company)+ is an open access article distributed under the Creative Commons Attribution License, which permits unrestricted use, distribution, and reproduction in any medium, provided the original work is properly cited. 